\title{
Waveform Design with Dual Ramp-Sequence for High-Resolution Range-Velocity FMCW Radar
}

\author{
Eugin Hyun ${ }^{1}$, Jong-Hun Lee ${ }^{1}$ \\ ${ }^{1}$ Advanced Radar Technology (ART) Lab., Daegu Gyeongbuk Institute of Science \& \\ Technology (DGIST), \\ 50-1, Sang-Ri, Hyeonpung-Myeon, Dalseong-Gun, Daegu, 711-873, Korea \\ braham@dgist.ac.kr
}

\begin{abstract}
Frequency modulated continuous wave (FMCW) radar using ramp-sequence is effective method for detecting the range and velocity of a target. For high-resolution range-velocity radar, wide bandwidth and numerous ramps must be generated. In that case, a high-speed sampling rate and a great deal of memory resources are required. Moreover, because fast Fourier Transform (FFT) is used to extract range-velocity profile, computational complexity also increases significantly. Thus, in order to overcome this problem, in this paper, we propose a new waveform with dual ramp-sequence with relatively low modulation slope compared to the conventional waveform. In the first ramp-sequence, the coarse range-profile and fine velocity-profile are extracted. In the second ramp-sequence, both high-resolution range-velocities are obtained, but ambiguous velocity may arise because of diminished maximum detectable-velocity values. This problem may be resolved by combining the results of both ramp-sequences. In this paper, we analysed the required sampling frequency of an analog-digital converter (ADC) and the amount of data memory required for typical and proposed methods. Moreover, from field programmable gate array (FPGA) based implementation results, we also estimated the processing times. Compared to that of the typical waveform, the reduction ratio for total processing time was $70 \%$.
\end{abstract}

Index Terms-High-resolution radar; FMCW radar; waveform design; human detection radar.

\section{INTRODUCTION}

Achieving active safety of recently developed intelligent vehicles requires that radar not only detect vehicles and pedestrians, but also recognize them [1]. One of the popular methods is based on the scattering distribution that appears on the range and Doppler spectra. Here, there is an extension in range and a point-shaped Doppler in the case of a vehicle and reverse characteristics in the case of a pedestrian [2]. For that, high-resolution range- and Doppler-profiles are required.

Moreover, these days, radars have provided a unique solution for sensing humans in law enforcement, security and surveillance operations, biomedical studies, and sports [3]. To detect and recognize humans, radar provides both high range-resolution and Doppler-resolution [3].

A frequency-modulated continuous-wave (FMCW) radar is very popular for range-Doppler processing because the

Manuscript received 17 November, 2015; accepted 28 February, 2016.

This work was supported by the DGIST R\&D Program of the Ministry of Science, ICT and Technology of Korea (16-RS-01). bandwidth of the received beat-signal decreases by less than a dozen $\mathrm{MHz}$ regardless of the transmitted bandwidth, and the complexity of the signal processing can be reduced, compared with that of conventional pulse Doppler radar [4][5]. Especially, FMCW radar based on a ramp-sequence with saw-tooth modulation shape is a very effective waveform to measure the target range and velocity simultaneously with high accuracy [6]-[7]. This is because the clutter is diminished in the zero Doppler-bin and then moving targets can easily be distinguished using 2D fast Fourier Transform (FFT) processing. In previous work [8], the waveform and signal processing was presented in detail (Fig. 1).

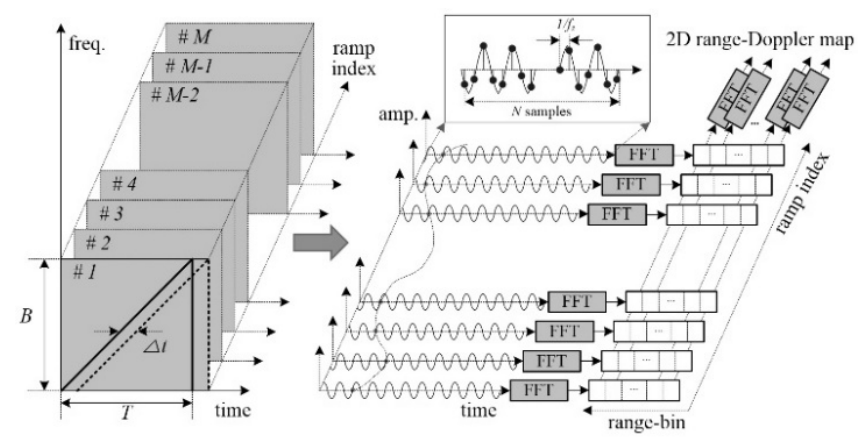

Fig. 1. Basic concept of 2D FFT processing in ramp-sequence based FMCW radar. Here, transmitted and received signals in the frequency-time domain are illustrated. Beat signals are digitalized and transferred into frequency domain every slow time. $B$ is the bandwidth, $T$ is the modulation period, $N$ is the number of samples per ramp, $M$ is the number of ramps, $f_{S}$ is analog-digital converter (ADC) sampling frequency, and $\Delta t$ is the two-way delay time of the received signal reflected from a single target.

In the ramp-sequence based FMCW radar, for high rangeresolution, the wide bandwidth of the transmitting signal is required, as shown in (1). Here, $\Delta R$ is range-resolution, $c$ is light velocity, and $B$ is the bandwidth. Therefore, in the case of wide bandwidth, the received beat-frequency increases

$$
\Delta R=\frac{c}{2 B}
$$

Moreover, if longer range detection is required, the beat-frequency is much higher such as (2). Here, $f_{b, \max }$ is maximum beat-frequency of the received signal, $R_{\max }$ is maximum detectable-range of target, and $c$ is light velocity. Moreover, the modulation-slope $\mu$ is expressed as $B / T$, which $B$ is the bandwidth and $T$ is the modulation period 


$$
f_{b, \max }=\mu\left(\frac{2 R_{\max }}{c}\right) .
$$

To decide the modulation period $(T)$ and the number of ramps $(N)$, we should consider the maximum detectable-velocity $V_{\max }$ and velocity resolution $(\Delta V)$, as in (3) and (4). Here, $f_{c}$ is the centeral frequency of carrier signal and $c$ is light velocity:

$$
\begin{gathered}
T=\frac{c}{4 f_{c} V_{\max }}, \\
\Delta V=\frac{c}{2 f_{c} T M} .
\end{gathered}
$$

According to (3) and (4), for radar with high-resolution velocity, numerous ramps must be generated. Moreover, when detectable-velocity is higher, $T$ should be shorter. This also causes beat-frequency to be higher, as shown in (2).

Therefore, the high-resolution range-velocity FMCW radar leads to raised maximum beat-frequency, and then ADC sampling frequency increases to provide successful digitalization of the beat-signal. For example, as shown in Fig. 2, the maximum beat-frequency is more than $30 \mathrm{MHz}$, when bandwidth $2 \mathrm{GHz}$, and the maximum detectable-velocity and range are $200 \mathrm{~km} / \mathrm{h}$ and $120 \mathrm{~m}$, respectively.

Because of that, the total computational complexity, including FFT and the memory size needed for data, also increases significantly. The lager the maximum detectable-range and velocity are, the more serious this is

These conditions eventually create such a burden for the hardware and software of the radar signal processor that the cost of the radar sensor rises.

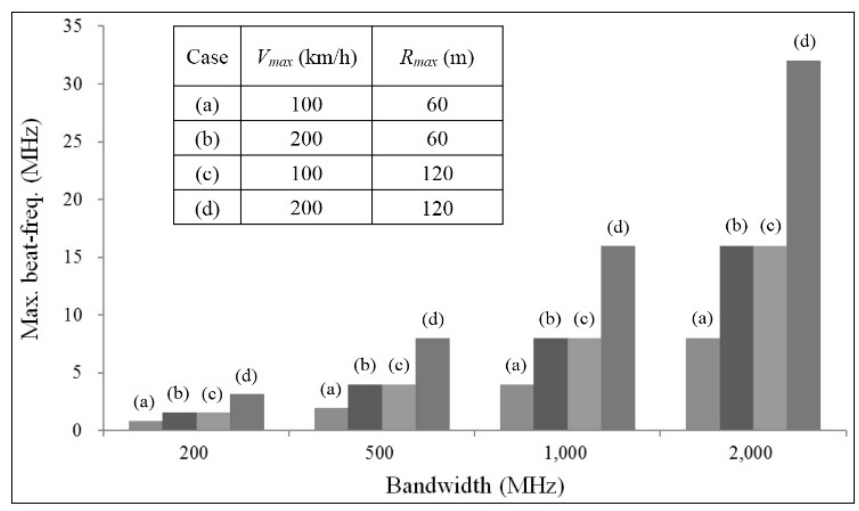

Fig. 2. Comparison of maximum beat-frequency according to four combinations of maximum detectable-range and velocity: Here, $\Delta \mathrm{R}$ is calculated as $0.75 \mathrm{~m}, 0.3 \mathrm{~m}, 0.15 \mathrm{~m}$, and $0.075 \mathrm{~m}$, based on B of $200 \mathrm{MHz}$, $500 \mathrm{MHz}, 1000 \mathrm{MHz}$, and $2000 \mathrm{MHz}$, respectively. Moreover, T is selected as $100 \mu \mathrm{s}$ and $50 \mu \mathrm{s}$ for $V \max$ of $100 \mathrm{~km} / \mathrm{h}$ and $200 \mathrm{~km} / \mathrm{h}$, respectively. Other radar parameters are described in Table II.

In this paper, we designed a waveform for high-resolution range-Doppler FMCW radar to overcome the problems mentioned above. We proposed a new waveform using a dual ramp-sequence with a low modulation-slope, compared to the typical waveform. In the first ramp-sequence, the coarse range-profile and fine velocity profile are extracted. In the second map, the high-resolution range-velocity is extracted, but ambiguous velocity can be obtained because the maximum detectable velocity is limited to its low value. However, by combining the results in both ramp-sequences, this problem is resolved and high-resolution range-velocity results are obtained with low complexity.

In Section II, we present the extraction procedure of a target 2D range-velocity map based on the newly design waveform. Section III presents the complexity analysis, simulations, and processing time estimation for the typical and proposed methods. The conclusions from our study are presented in Section IV.

\section{WAVEFORM DESIGN USING DUAL RAMP-SEQUENCE}

First, a typical FMCW waveform is illustrated in Fig. 3 (a). In addition, we also present a new waveform designed with a dual ramp-sequence for high-resolution range-Doppler FMCW radar, shown in Fig. 3(b).

The designed waveform is divided into two ramp-sequences. Compared to the typical waveform, the modulation-slope is reduced to $\mu / K$. Here, we define $K$ as the modulation-slope reduction factor. The detailed description of the waveform is as follows:

1) RS (Ramp-sequence) \#1:

1. The waveform with narrow bandwidth $(B / K)$ is transmitted, thus range-resolution decreases as $\Delta R / K$.

2. The maximum detectable velocity and velocity resolution are equal to those of a conventional waveform, because the modulation period and number of ramps are the same.

2) $R S$ (Ramp-sequence) \#2:

1. While the wide bandwidth $(B)$ is used, the modulation period becomes longer as $T \times K$ and the number of ramps decreases as $M / K$.

2. Thus, range-resolution is extracted as $\Delta R$, but the maximum detectable-velocity decreases as $V_{\max } / \mathrm{K}$. As a result, velocity-resolution can be still maintained as $\Delta V$.

(a)

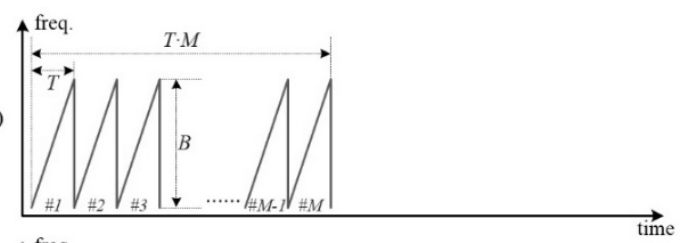

(b)

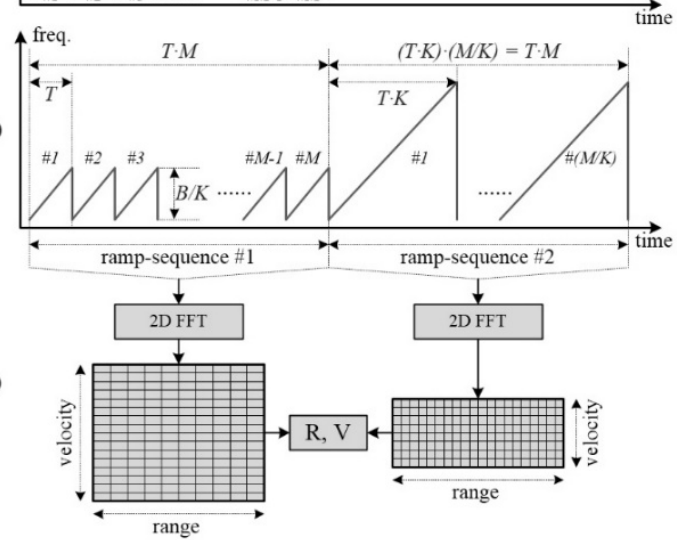

Fig. 3. Ramp-sequence based FMCW radar waveform: a - typical waveform with modulation-slope $\mu ; \mathrm{b}$ - proposed waveform comprising two ramp-sequences with $\mu / K ; \mathrm{c}-2 \mathrm{D}$ FFT-based target detection scheme in the proposed waveform. 
So far, based on the information described, range-velocity maps extracted by the typical waveform and the two ramp-sequences of the proposed method are illustrated in Fig. 4(a)-Fig. 4(c), respectively. In this example, we assume that a single moving target with multiple scattering points exists within detectable-range $\left(R_{\max }\right)$ and velocity $\left(V_{\max }\right)$.

In the typical method, as illustrated in Fig. 4(a), the target with high-resolution range $(\Delta R)$ and velocity $(\Delta V)$ are detected as 12 cells.

In the first ramp-sequence of the proposed method, a target is detected with only 6 cells in the low-resolution range domain, shown in Fig. 4(b). In that case, the velocity grid is still high-resolution. In this step, we can obtain the coarse range-profile and fine velocity-profile of the target.

In the second ramp-sequence, we can extract both fine range- and velocity-profiles comprising 12 cells, shown in Fig. 4(c). However, we should consider the aliasing problem of the detected velocity. That is, when the velocity of a moving target is more than $V_{\max } / K$, the detected Doppler frequency of this target is placed in the wrong frequency domain.

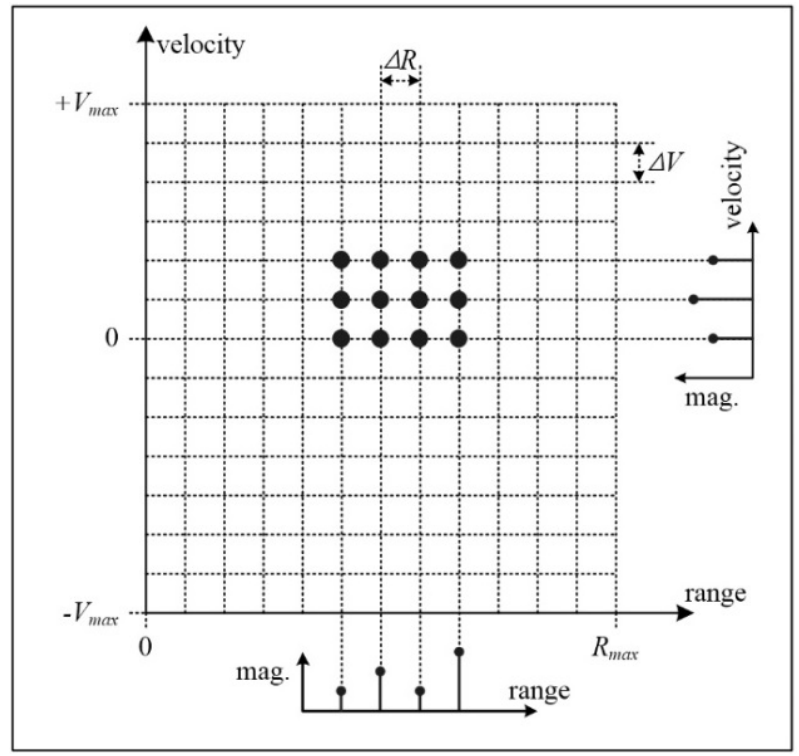

a)

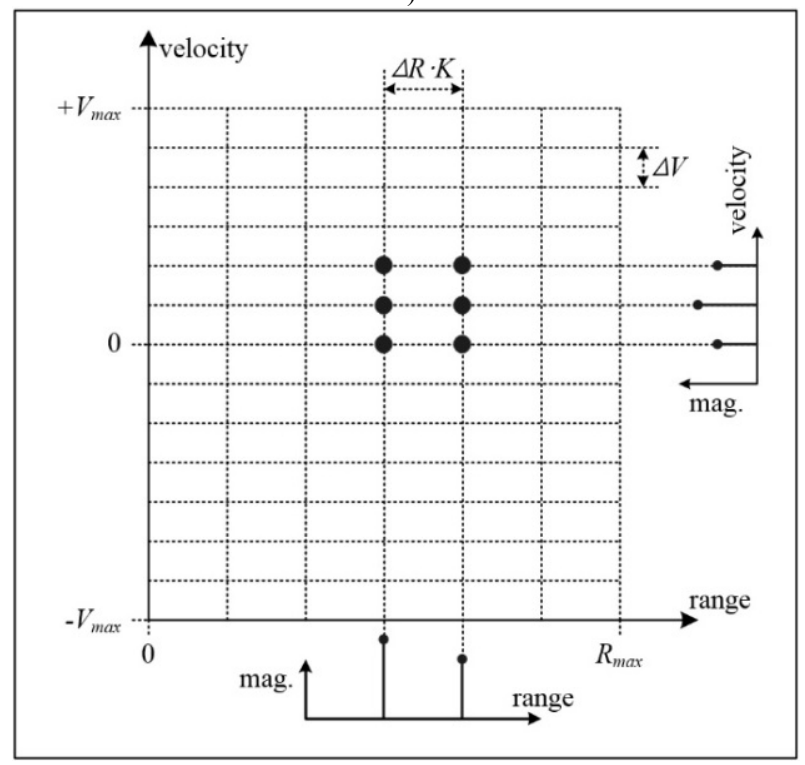

b)

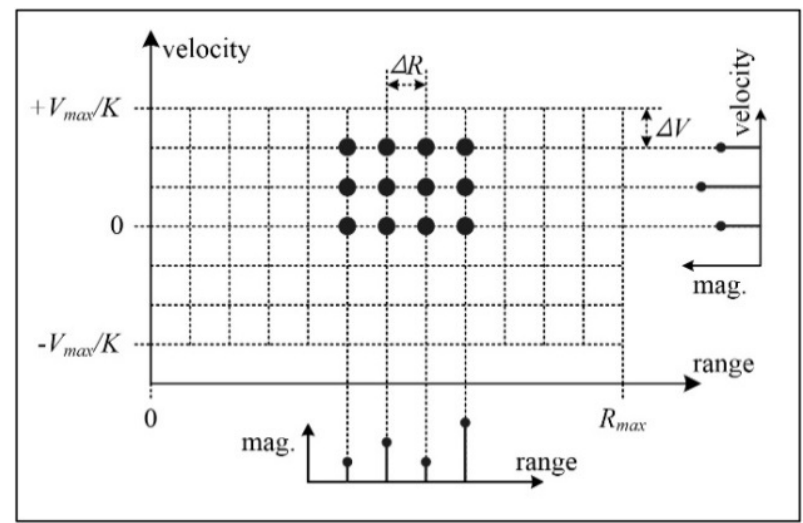

c)

Fig. 4. Illustration of the extracted range-velocity map: a - example of typical method; $b$ - example of the first ramp-sequence in the proposed waveform; c - example of the second in the proposed method. Here, red circles present the detected scattering points of echo signal reflected from single moving target.

In order to explain this problem in detail, we present the example of one moving target with $f_{D 1 \text {,target }}$ Doppler frequency, shown in Fig. 5. Here, the x-axis indicates Doppler frequency and the $\mathrm{y}$-axis is magnitude.

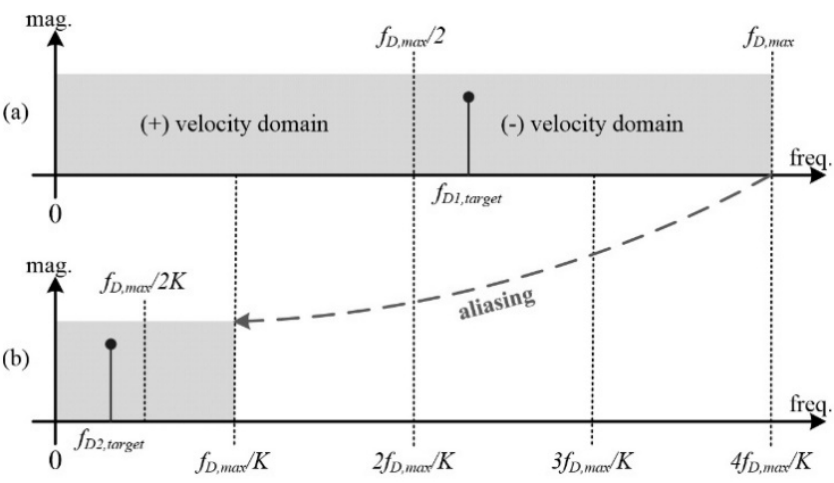

Fig. 5. Example to explain aliasing on the Doppler frequency domain of the proposed waveform: a - case of first ramp-sequence; b - case of second ramp-sequence. Here, $f_{D, \max }$ is the maximum Doppler frequency to be detected, and $f_{D 1 \text {,target }}$ and $f_{D 2 \text {,target }}$ are the detected Doppler frequencies in each ramp-sequence. Moreover, in the example, $K$ is 4 .

In the result of the first ramp-sequence of Fig. 5(a), the Doppler frequency of the target can be normally detected as $f_{D 1, \text { target }}$. Here, the Doppler frequencies on 0 to $\left(f_{D, \text { max }} / 2\right)$ and $\left(f_{D, \text { max }} / 2\right)$ to $f_{D \text {, max }}$ are mapped on the corresponding positive and negative velocities, respectively. Thus, in this example, we can determine that the detected target has negative velocity, which means the target is coming toward the radar. Here, the maximum Doppler frequency $\left(f_{D, \text { max }}\right)$ is calculated with determination of the detectable-velocity $\left(V_{\max }\right)$ of (3).

However, in the example of the second ramp-sequence with $K=4$, the Doppler frequency of the target appears as within 0 to $\left(f_{D, \max } / K\right)$, due to aliasing, shown in Fig. 5(b).

In reference [9], the ambiguous Doppler frequency $f_{D 2, \text { target }}$ can be express in (5), where factor $q$ is 0 to $(K-1)$. Here, because we know already $f_{D 1 \text {,target }}$ and $f_{D, \max } / K$, we can determine $q$; in this example, $q$ is ' 2 ' 


$$
f_{D 2, \text { target }}=f_{D 1, \text { target }}-q \frac{f_{D, \max }}{K} .
$$

Therefore, based on cells of range-velocity extracted from both ramp-sequences, we can obtain the same high-resolution range-velocity as the typical method does.

\section{SimULATION AND IMPLEMENTATION ReSUltS}

\section{A. Complexity Analysis}

Based on the contents in Section II, we analysed the hardware and processing complexity for the typical method and the proposed waveform, shown in Table I. The analysis details are described as follows:

1) $A D C$ sampling frequency:

1. Based on Nyquist sampling theory, the ADC sampling rate is higher than at least twice the maximum beat-frequency.

2. For FMCW radar, as shown in (5), because the maximum beat-frequency is determined by $\mu$ (modulation-slope), the ADC sampling frequency required in the proposed waveform is $K$ times lower than that of typical method.

\section{2) Data memory:}

1. For the proposed waveform, because dual ramp-sequences are used, the total data acquisition time is twice that of conventional method (Fig. 3).

2. Even so, the ADC sampling frequency of the proposed method is $K$ times lower, and the data memory used for the proposed method is $K / 2$ times lower than that needed for the typical method.

TABLE I. COMPLEXITY ANALYSIS RESULTS.

\begin{tabular}{|c|c|c|c|c|}
\hline \multicolumn{2}{|c|}{} & $\begin{array}{c}\text { ADC sampling } \\
\text { frequency }\end{array}$ & $\begin{array}{c}\text { Data } \\
\text { memory }\end{array}$ & FFT complexity \\
\hline \multicolumn{2}{|c|}{ Typical method (a) } & $>2 \mu \times \frac{2 R_{\max }}{c}$ & $N \times M$ & $\begin{array}{l}N \times \log _{2} N \times M+ \\
+M \times \log _{2} M \times M\end{array}$ \\
\hline $\begin{array}{c}\text { Proposed } \\
\text { method } \\
\text { (b) }\end{array}$ & $\mathrm{RS} \mathrm{\# 1}$ & $>2 \mu \times \frac{\mu}{K} \times \frac{2 R_{\max }}{c}$ & $\frac{N}{K} \times M$ & $\begin{array}{l}\frac{N}{K} \times \log _{2} \frac{N}{K} \times M+ \\
+M \times \log _{2} M \times \frac{N}{K}\end{array}$ \\
\hline $\begin{array}{c}\text { Reduction ratio } \\
\text { (b)/(a) }\end{array}$ & $\mathrm{KS} \# 2$ & $>2 \mu \times \frac{\mu}{K} \times \frac{2 R_{\max }}{c}$ & $N \times \frac{M}{K}$ & $\begin{array}{l}N \times \log _{2} N \times \frac{M}{K}+ \\
+\frac{M}{K} \times \log _{2} \frac{M}{K} \times N\end{array}$ \\
\hline
\end{tabular}

\section{3) FFT complexity:}

1. While two FFTs are processed to generate range-velocity in the typical method, four FFT processing steps are conducted for the two ramp-sequences of the proposed waveform. However, because the total number of data samples is reduced, total computational complexity also decreases.

2. Based on the equations in Table I, we estimated the computational complexity of $K$, shown in Fig. 6. Here, all results are normalized using the complexity of the typical method. In the case with $K$ of 4 , the FFT complexity decreases to less than $50 \%$ of the typical method. Moreover, we also see that the complexity is dramatically reduced, when $K$ is larger.

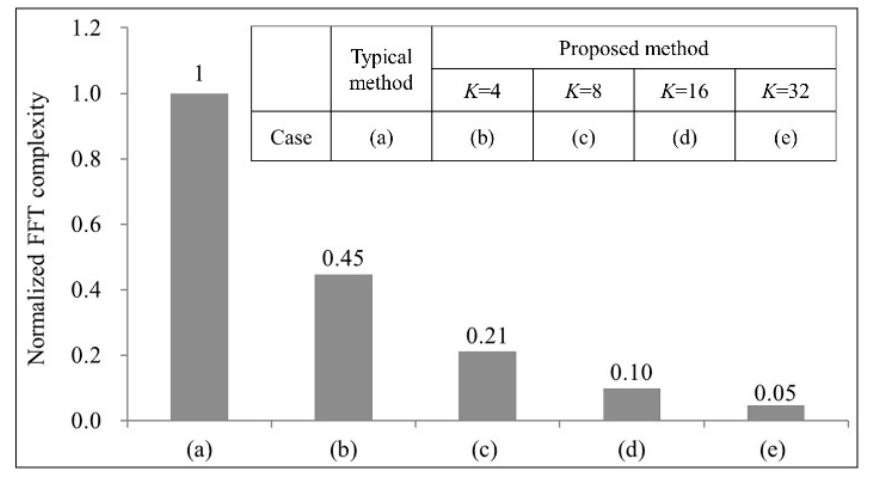

Fig. 6. Comparison of FFT complexity based on modulation-slope reduction factor $(\mathrm{K})$ : Here, the applied radar parameters are described in Table II.

\section{B. Simulation Results}

For simulations and implementations, we determined the optimal radar parameters for waveform and FFT processing. The maximum detectable-range and velocity were determined to be $60 \mathrm{~m}$ and $100 \mathrm{~km} / \mathrm{h}$. With the typical waveform, for the high-resolution range and velocity, the bandwidth and the number of ramps were determined to be $2 \mathrm{GHz}$ and 256. For the proposed method, we selected the modulation-slope reduction factor $(K)$ of ' 4 '. Based on $K$, all parameters of the proposed method are calculated according to the concepts in Fig. 3.

The ADC sampling frequencies selected for the typical and proposed methods were $20.48 \mathrm{MHz}$ and $5.12 \mathrm{MHz}$, respectively. The value of the proposed method seems to that it is more reasonable for minimizing the burden of hardware design for implementation of signal processing.

Based on the parameters in Table II, we extracted 2D profiles of pseudo-targets. In this simulation, we assumed that the target had multiple scattering points on the range-velocity domains. Figure 7(a) shows seven pairs with range $(\mathrm{m})$ and velocity $(\mathrm{km} / \mathrm{h}):(48.78,88.77),(49.00,89.65)$, (49.15, 89.65), (49.07, 90.53), (49.22, 90.53), (48.85, 89.65), and $(48.93,90.53)$.

TABLE II. RADAR PARAMETERS USED.

\begin{tabular}{|c|c|c|c|c|}
\hline \multirow{2}{*}{ System parameters } & \multirow{2}{*}{$\begin{array}{c}\text { Symbols } \\
\text { (Unit) }\end{array}$} & $\begin{array}{c}\text { Typical } \\
\text { method }\end{array}$ & \multicolumn{2}{|c|}{$\begin{array}{c}\text { Proposed method } \\
(K=4)\end{array}$} \\
\cline { 4 - 5 } & & RS \#1 & RS \#2 \\
\hline Centre frequency & $f_{\mathrm{c}}(\mathrm{GHz})$ & 24 & 24 & 24 \\
\hline Bandwidth & $B(\mathrm{GHz})$ & 2 & 0.5 & 2 \\
\hline Modulation period & $T(\mathrm{us})$ & 100 & 100 & 400 \\
\hline Number of ramps & $M(\mathrm{set})$ & 256 & 256 & 64 \\
\hline ADC sampling rate & $f_{s}(\mathrm{MHz})$ & 20.48 & 5.12 & 5.12 \\
\hline Range-FFT point & $N(\mathrm{point})$ & 2048 & 512 & 2048 \\
\hline Doppler-FFT point & $M(\mathrm{point})$ & 256 & 256 & 64 \\
\hline Max. detectable-range & $R_{\max }(\mathrm{m})$ & 60 & 60 & 60 \\
\hline Max. detectable-velocity & $V_{\max }(\mathrm{km} / \mathrm{h})$ & 100 & 100 & 25 \\
\hline Range resolution & $\Delta R(\mathrm{~m})$ & 0.075 & 0.300 & 0.075 \\
\hline Velocity resolution & $\Delta V(\mathrm{~km} / \mathrm{h})$ & 0.879 & 0.879 & 0.879 \\
\hline
\end{tabular}


In Fig. 7(b), the high-resolution range-velocity profiles are extracted based on the typical waveform. Figure 7(c) shows the coarse range-profile and fine velocity-profile in the first ramp-sequence of the proposed waveform. In Fig. 7(d), it was found that the range-velocity map had high-resolution, but also that the velocity values were located in the wrong position due to Doppler frequency aliasing. However, this could be revised using velocity the information obtained in Fig. 7(c).

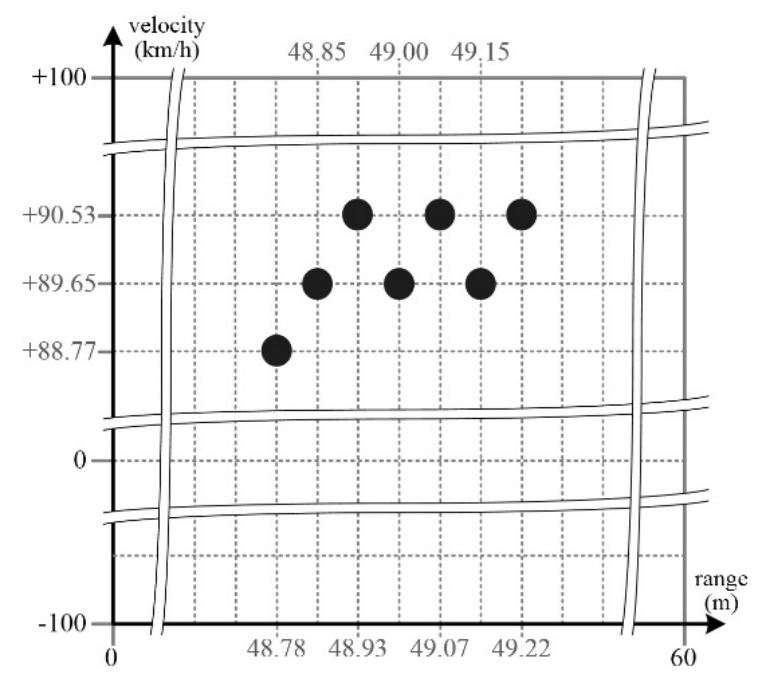

a)
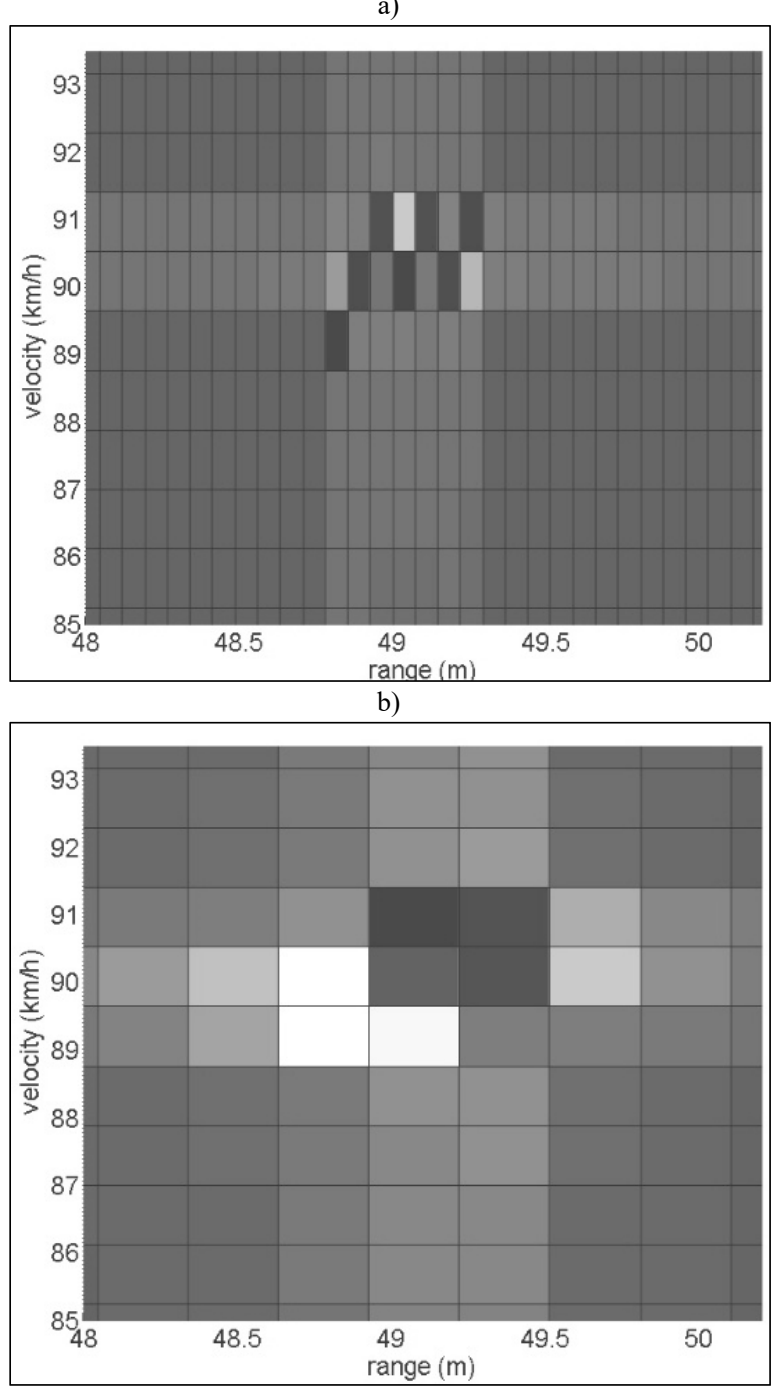

c)

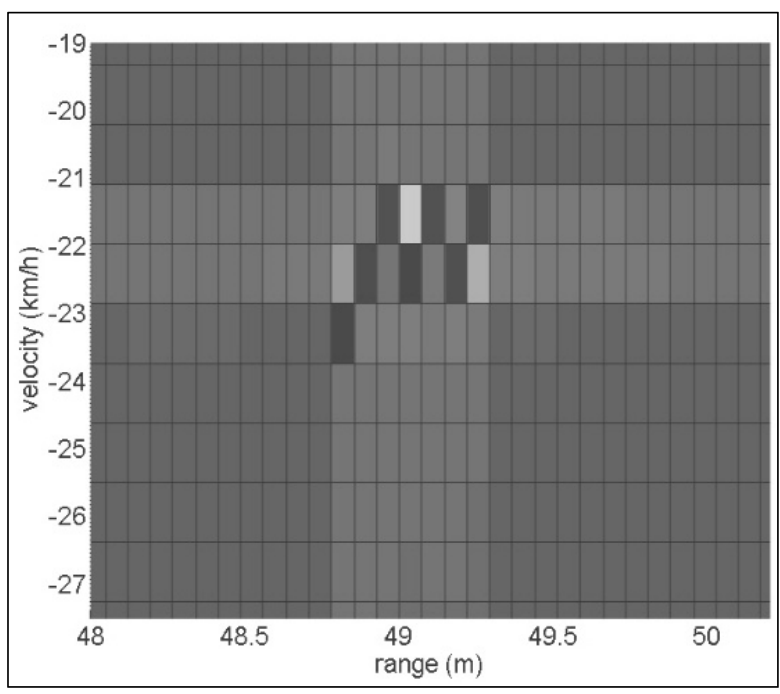

d)

Fig. 7. Simulation scenario and results: a - multiple scattering points of a pseudo-target in the range-velocity domain; $b$ - result from the typical method; $\mathrm{c}$ - result in the first sequence ramp of the proposed waveform; $\mathrm{d}-$ result in the second sequence.

\section{Implementation Results}

The total processing time is measured based on implementation of a field programmable gate array (FPGA). For high-speed processing, we employ data-routing-based pipelined and parallel architecture for the multi-channel FMCW radar designed in the previous work [8].

Figure 8 shows the signal processing architecture used to extract 3D (range-velocity-angle) profile for the typical and proposed methods. First, the window function and FFT were applied for range- and Doppler-processing. Moreover, in order to extract angle spectra, the digital beam forming (DBF) technique was used on eight receiving channels. In this paper, we did not consider the target decision blocks such as the CAFR (constant false alarm rate) detector, peak finding, or thresholding.

With the proposed method, because a dual ramp-sequence is used, this $3 \mathrm{D}$ profile extraction procedure is repeated twice for each sequence.

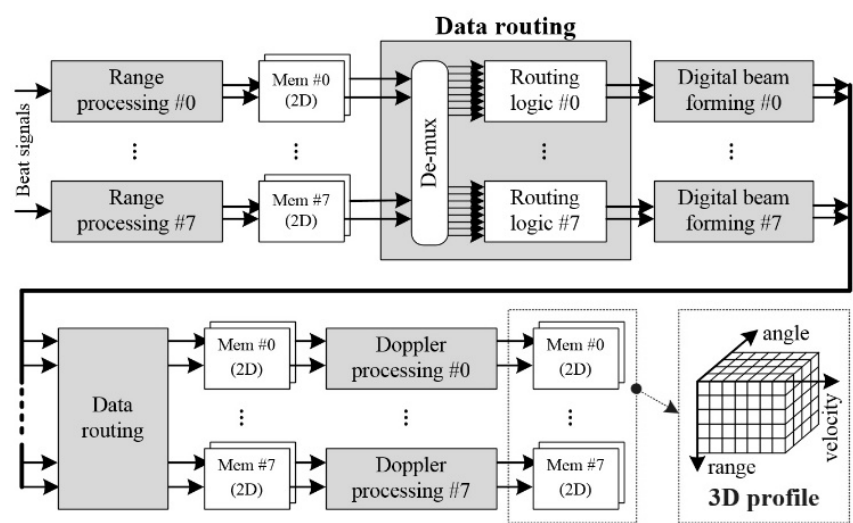

Fig. 8. Range-velocity-angle map extraction architecture using the data-routing-based pipelined and parallel architecture designed in the previous work [8].

The range-velocity-angle map extraction processing was implemented in Xilinx Viretex-5 XC5VLX330 using verilog hardware description language (HDL). Figure 9 presents a block diagram of FPGA-based firmware structure.

The ADC digitalizes beat signals of eight channels 
simultaneously. Next, in the Des blocks, the received serial bit streams are de-serialized into words. Finally, the Sync blocks synchronize the words with the transmitting trigger.

In 3D (range-velocity-angle) profile extraction blocks, the FFTs were implemented using Xilinx LogiCORE ${ }^{\mathrm{TM}}$ IP Fast Fourier Transform v7.0, which is based on Radix-2 Burst I/O architecture with a streaming 16-bit data length. In addition, DBF was also implemented based on Xilinx LogiCORE ${ }^{\mathrm{TM}} \mathrm{IP}$ Multiplier v11.2.

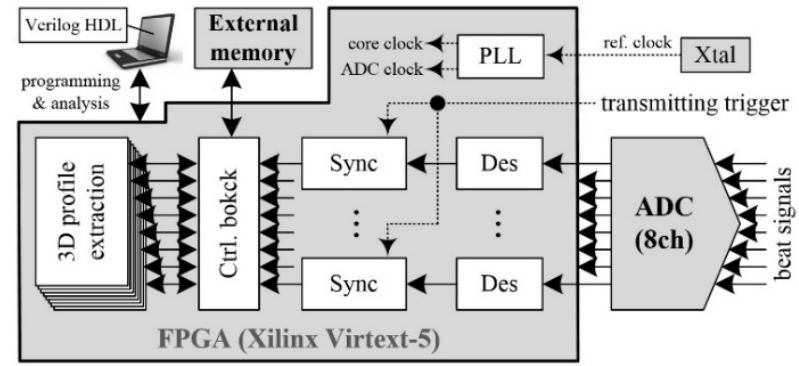

Fig. 9. Simplified FPGA-based firmware structure to implement 3D profile extraction processing.

The implementation summary for the typical and proposed waveforms was presented in Table III. Here, one slice contains four LUTs and four flip-flops. One DSP48E device for fast calculations consists of a multiplier, an adder, and an accumulator. In addition, one embedded memory block is 36 Kbits in size [10].

Compared to the typical waveform, in the proposed waveform, while the required slice registers and slice LUTs were similar, embedded memory resources was more consumed. This is because the additional FFT blocks are needed for the both sequences of proposed waveform.

On the other hand, as mentioned in Table I, the needed external memory of the typical method is two times higher than that required for the proposed method.

TABLE III. IMPLEMENTATION SUMMARY FOR TYPICAL AND PROPOSED WAVEFORMS BASED SIGNAL PROCESSING.

\begin{tabular}{|c|c|c|c|c|}
\hline \multicolumn{2}{|c|}{ Functions } & $\begin{array}{l}\text { Available } \\
\text { resources }\end{array}$ & Typical method & Proposed method \\
\hline \multirow{2}{*}{$\begin{array}{c}\text { FPGA } \\
\text { internal } \\
\text { resources }\end{array}$} & Slice registers & 51,840 & 15,778 & 18,732 \\
\cline { 2 - 5 } & Slice LUTs & 51,840 & 23,136 & 25,881 \\
\cline { 2 - 5 } & Embedded memory & 288 & 136 & 232 \\
\hline \multicolumn{2}{|r|}{ External memory } & 192 & 160 & 160 \\
\hline
\end{tabular}

TABLE IV. TOTAL PROCESSING TIME OF TYPICAL AND PROPOSED WAVEFORMS BASED SIGNAL PROCESSING.

\begin{tabular}{|c|c|c|c|}
\hline \multirow{2}{*}{ Functions } & \multicolumn{3}{|c|}{ Processing Time (ms) } \\
\cline { 2 - 4 } & $\begin{array}{c}\text { Typical } \\
\text { method }\end{array}$ & RS \#1 & RS \#2 \\
\cline { 2 - 4 } & 25.60 & 25.60 & 25.60 \\
\hline $\begin{array}{c}\text { ADC data } \\
\text { acquisition }\end{array}$ & 332,54 & 32.34 & 23.44 \\
\hline $\begin{array}{c}\text { Algorithm } \\
\text { processing }\end{array}$ & 358.14 & \multicolumn{2}{|c|}{106.98} \\
\hline Total & \multicolumn{3}{|c|}{} \\
\hline
\end{tabular}

Next, the total processing times were estimated, as shown in Table IV. It can be seen that the typical and proposed methods required approximately $358.14 \mathrm{~ms}$ and $106.98 \mathrm{~ms}$, respectively. We select the FPGA internal core clock frequency of $100 \mathrm{MHz}$, because the maximum frequency in synthesis results was reported as $103.036 \mathrm{MHz}$.

Compared to the typical method, the total processing in the proposed waveform was reduced by more than $70 \%$, even though the ADC data logging consumed twice as much time.

In these results, because the data transfer time from/to external memory was also not considered, the real processing time could be longer for both methods.

\section{CONCLUSIONS}

For high-resolution range-velocity of the typical ramp-sequence-based FMCW waveform; wide bandwidth, shorter modulation period, and numerous ramps are required. However, this leads to increased utilization of hardware resources and to increased computational complexity.

In this paper, we proposed a new waveform with dual ramp-sequence. Because of the low modulation-slope, the required $\mathrm{ADC}$ sampling rate, size of memory used for data, and FFT complexity, could decrease. In particular, when the modulation-slope reduction was greater, it was proved that they could be dramatically reduced through analysis results.

In this paper, typical and proposed methods were implemented in Xilinx Vertex-5 FPGA to estimate total processing time. In the results, the processing time of the new waveform was $70 \%$ less than of the conventional method.

Therefore, the proposed waveform could be useful for applications with high-resolution range and velocity profiles.

\section{REFERENCES}

[1] D. Geronimo, A. M. Lopez, A. D. Sappa, T. Graf, "Survey of pedestrian detection for advanced driver assistance systems", IEEE Trans. Pattern Analysis and Machine Intelligence, vol. 32, no. 7, pp. 1239-1258, 2010. [Online]. Available: http://dx.doi.org/10.1109/ TPAMI.2009.122

[2] S. Heuel, H. Rohling, "Pedestrian classification in automotive radar system", IEEE Int. Radar Symposium, 2012, pp. 39-44. [Online]. Available: http://dx.doi.org/10.1109/irs.2012.6233285

[3] S. S. Ram, A. Majumdar, "High-resolution radar imaging of moving humans using doppler processing and compressed sensing", IEEE Trans. Aerospace and Electronic Systems, vol. 51, no. 2, pp. 12791287, 2015. [Online]. Available: http://dx.doi.org/10.1109/TAES. 2014.140481

[4] M. A. Richards, Fundamentals of Radar Signal Processing. McGraw-Hill, 2005.

[5] S. Miyahara, "New algorithm for multiple object detection in FM-CW radar”, SAE Technical Paper Series, 2004.

[6] M. Kronauge, C. Schroeder, H. Rohling, "Radar target detection and doppler ambiguity resolution", Radar Symposium, 2010, pp. 1-4.

[7] C. Schroeder, H. Rohling, "X-band FMCW radar system with variable chirp duration", IEEE Radar Conf., 2010, pp. 1255-1259. [Online]. Available: http://dx.doi.org/10.1109/radar.2010.5494425

[8] E. G. Hyun, S. D. Kim, J. H. Choi, D. J. Yeom, J. H. Lee, "Parallel and pipelined hardware implementation of radar signal processing for an FMCW multi-channel radar", Elektronika ir Elektrotechnika, vol. 21, no. 2, pp. $65-71,2015$. [Online]. Available: http://dx.doi.org/10.5755/ j01.eee.21.2.7606

[9] M. Kronauge, H. Rohling, "New chirp sequence radar waveform", IEEE Trans. Aerospace and Electronic Systems, vol. 50, no. 4, pp. 2870-2877, 2014. [Online]. Available: http://dx.doi.org/10.1109/ TAES.2014.120813

[10] Virtex-5 Family Overview (DS100). [Online]. Available: http://www.xilinx.com/support/documentation/data_sheets/ds100.pdf 